\title{
All polyethylene tibia - results of functional and radiological outcome at 5 years follow-up
}

\author{
Digen Patel, Kunal Ajitkumar Shah*, Mohan Madhav Desai, Swapneel S. Shah
}

Department of Orthopaedics, Seth G. S. Medical College and KEM Hospital, Mumbai, Maharashtra, India

Received: 06 March 2020

Revised: 07 June 2020

Accepted: 09 June 2020

\section{*Correspondence: \\ Dr. Kunal Ajitkumar Shah, \\ E-mail: kunalajitshah@gmail.com}

Copyright: (c) the author(s), publisher and licensee Medip Academy. This is an open-access article distributed under the terms of the Creative Commons Attribution Non-Commercial License, which permits unrestricted non-commercial use, distribution, and reproduction in any medium, provided the original work is properly cited.

\begin{abstract}
Background: Earlier, all-polyethylene tibial (APT) components were the only option available for TKA. APT components improved over years and have shown results similar or superior to MBT components with respect to survivorship and rate of complications. This study aimed to assess the functional and radiological outcome of TKA with APT components with 5 years follow-up.

Methods: This longitudinal study was conducted in a tertiary care center in Mumbai in year 2018. All patients who underwent TKA with APT components during the year 2012 were included in the study. Total of 147 knees were operated in 120 patients. Ninty patients (111 knees) followed up at 5 years. Preoperative and postoperative assessment of patients was done for function, pain and radiological outcome. Functional scores used were Knee society score, WOMAC osteoarthritis index, Bristol score and Oxford knee score. Visual analogue scale (VAS) score was used for pain assessment. Pre and postoperative X-rays were done and component alignment angles $(\alpha, \beta, \gamma$ and $\delta)$ were calculated.

Results: Statistically significant difference was found in pre and postoperative values of VAS score, knee society score, WOMAC osteoarthritis index, Bristol score and Oxford knee score ( $\mathrm{p}$ value $<0.001$ ). Component alignment angles were found to be same pre and postoperatively. None of the patients had radiologic evidence of loosening of implants.

Conclusions: APT components have good to excellent functional outcome and good radiological outcome at 5 years. They are safe in view of radiologic component migration at 5-year follow-up.
\end{abstract}

Keywords: All polyethylene tibia, Component alignment, Metal back tibia

\section{INTRODUCTION}

Tibial components for total knee arthroplasty (TKA) have come a long way with improved materials and better design. Earlier, all-polyethylene tibial (APT) components were the only option available for TKA. ${ }^{1}$ Tibial components were made of ultra-high-molecular-weight polyethylene (UHMWPE). One of the extensively studied APT designs was University of California at Irvine (UCI) total knee prosthesis. Its tibial component was U-shaped with thickness of 5 to $7.5 \mathrm{~mm}$. Early failure of this component was attributed to its design and operative technique. Other similar design components also had low conformity, lower tibial plateau coverage and poor survivorship leading to their failure. Due to unfavorable results from these studies, surgeons shifted in favor of MBT components and the use of APT components phased out. ${ }^{2-4}$

The MBT components improved theoretical load transfer (especially during asymmetrical load transfer), fixation, provided intraoperative flexibility for soft tissue balancing, options to use augments or stem extenders and allowed isolated liner exchange in cases of polyethylene 
wear. But MBT come with their own set of disadvantages viz. backside wear, loosening, reduced polyethylene thickness with same amount of bone resection and increased tensile stress at cement bone interface. ${ }^{1}$

Over the years, APT components have improved, especially in the development of better polyethylene materials, biomechanically better implant design and improved surgical technique. They have shown results similar or superior to MBT components with respect to survivorship and rate of complications. ${ }^{5-9}$ These modern APT components have improved mechanical properties, edge-loading tolerance and survivorship. ${ }^{10}$ Other advantages are absence of backside wear and locking mechanism failures, cost-efficiency and usage of thicker polyethylene with decreased bone resection. ${ }^{1}$ This longitudinal study was therefore taken up with the aim to assess the functional and radiological outcome of TKA with APT components with 5 years follow-up.

\section{METHODS}

This longitudinal study was conducted during January 2012 to December 2018 in an orthopedic outpatient department (OPD) of a tertiary care center in a metropolitan city after obtaining ethical clearance from the institutional ethics committee.

\section{Inclusion criteria}

All patients who underwent TKA during January 2012 to December 2012 with APT component were included.

\section{Exclusion criteria}

Patients who have undergone TKA with other type of prosthesis and those who didn't give consent for study were excluded.

All the patients who underwent all poly TKA in 2018 were included in the study. A total of 147 knees were operated in 120 patients. Twenty-seven patients under went bilateral TKA. Seven patients refused to participate in the study. Hence 113 patients were enrolled in the study. Twentythree patients were lost to follow-up. Therefore 90 patients followed up after 5 years, out of which 21 patients has undergone bilateral TKA. In all, 111 knees were included in the study.

Pre and post-operative assessment of patient were done for function and pain. Functional assessment was done by knee scoring systems and pain assessment was done using visual analogue scale (VAS). Pre and postoperative scoring used were knee society score, WOMAC osteoarthritis index, Bristol knee score and Oxford knee score.

Preoperative assessment of function and pain was done. A single senior surgeon at this study center performed all the knee replacements by medial para-patellar approach.
According to protocol, all patients were mobilized full weight bearing from day one post-operatively. At 5-year follow-up repeat knee functional scoring and VAS scoring was done. Radiological investigation in the form of X-ray knee (anteroposterior and lateral views) was done both immediate postoperatively and at 5-year follow-up. Alignment of the TKA components were calculated on the $\mathrm{X}$-ray as per American knee society recommendations. Angles were labeled as alpha $(\alpha)$, beta $(\beta)$, gamma $(\gamma)$ and delta $(\delta)$ angles which determine femoral component varus/valgus, tibial component varus/valgus, femoral component flexion/extension and tibial slope respectively. ${ }^{11}$ (Table 1, Figure 1).

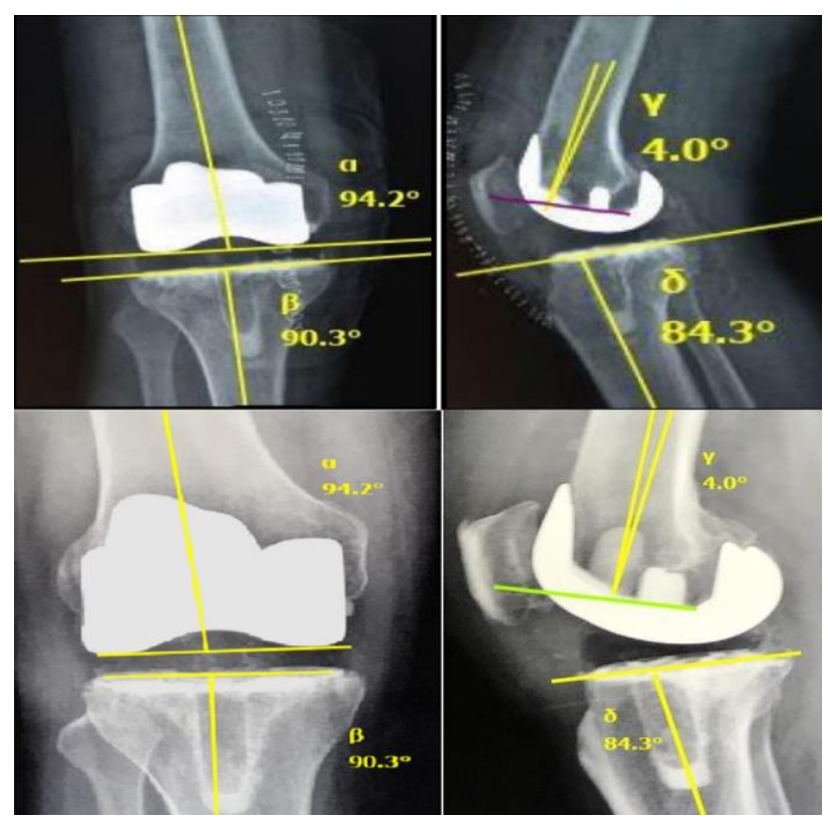

Figure 1: Method for calculation of component alignment angles on anteroposterior and lateral $\mathrm{X}$ rays both immediate and 5 year postoperative.

Table 1: Description of angles used to measure the implant position in coronal and sagittal plane.

\begin{tabular}{|c|c|}
\hline Angles & Description \\
\hline$\alpha$ & $\begin{array}{l}\text { The medial angle between a line drawn } \\
\text { parallel with the femoral component } \\
\text { condyles and the anatomical axis of the } \\
\text { femur. }\end{array}$ \\
\hline$\beta$ & $\begin{array}{l}\text { The medial angle between a line drawn } \\
\text { parallel to the tibial component on the } \\
\text { AP X-ray and the anatomical axis of } \\
\text { the tibia. }\end{array}$ \\
\hline$\gamma$ & $\begin{array}{l}\text { Sagittal proximal angle between a line } \\
\text { drawn perpendicular to the distal } \\
\text { cement interface of the femoral } \\
\text { component and the femoral anatomical } \\
\text { axis in the lateral X-ray. }\end{array}$ \\
\hline$\delta$ & $\begin{array}{l}\text { Sagittal posterior angle between a line } \\
\text { drawn parallel to the tibial component } \\
\text { and the anatomical tibial axis in the } \\
\text { lateral X-ray. }\end{array}$ \\
\hline
\end{tabular}


Data collected from all the patients were grouped into preoperative and post-operative data. Comparison between variables were made using various statistical tests. Mean value and standard deviation of follow up period, age, preoperative and postoperative pain, WOMAC osteoarthritis knee score, knee society score, oxford knee score, Bristol knee score were calculated. Data was statistically described in terms of mean $( \pm \mathrm{SD})$, frequencies (number of cases) and percentages wherever appropriate. Pre- and post-comparison of various scores were done using Wilcoxon's Sign Rank test. A probability value (p value) less than 0.05 was considered statistically significant.

\section{Statistical analysis}

All statistical calculations were done using computer programs Microsoft excel 2016 (Microsoft Corporation, NY, USA) and SPSS (statistical package for the social science; IBM SPSS statistics) version 21.

\section{RESULTS}

This longitudinal study of 111 knees in 90 patients of TKA (21 patients underwent bilateral TKA) yielded the following results.

Table 2: Pre-operative and post-operative values of VAS scores and functional knee scores of study participants.

\begin{tabular}{|c|c|c|c|c|}
\hline \multirow{2}{*}{ Score } & \multirow{2}{*}{$\mathbf{N}=$ number of knees } & Pre-operative & Post-operative & \multirow{2}{*}{$P$ value * } \\
\hline & & Mean \pm SD & Mean \pm SD & \\
\hline VAS (visual analogue scale) score & 111 & $6.83 \pm 0.70$ & $1.69 \pm 0.67$ & $<0.001$ \\
\hline WOMAC osteoarthritis index & 111 & $48.47 \pm 4.78$ & $14.11 \pm 2.44$ & $<0.001$ \\
\hline Oxford knee score & 111 & $41.81 \pm 3.71$ & $13.06 \pm 1.09$ & $<0.001$ \\
\hline Bristol knee score & 111 & $21.03 \pm 2.46$ & $41.44 \pm 1.61$ & $<0.001$ \\
\hline Knee society objective knee score & 111 & $54.06 \pm 5.33$ & $88.75 \pm 4.47$ & $<0.001$ \\
\hline Knee society functional score & 111 & $41.03 \pm 2.18$ & $91.62 \pm 4.42$ & $<0.001$ \\
\hline
\end{tabular}

*Test used is Wilcoxon sign rank test.

Table 3: Angles $(\alpha, \beta, \gamma$ and $\delta)$ in immediate post-operative and follow-up radiographs.

\begin{tabular}{|llllll|}
\hline Variable & Mean \pm SD & Range & $\mathbf{2 5}^{\text {th }}$ percentile & $\mathbf{5 0}^{\text {th }}$ percentile & $\mathbf{7 5}^{\text {th }}$ percentile \\
\hline $\boldsymbol{\alpha}$ & $95.29 \pm 1.43$ & 6.0 & 94.0 & 96.0 & 96.0 \\
\hline $\boldsymbol{\beta}$ & $90.00 \pm 0.85$ & 4.0 & 90.0 & 90.0 & 90.0 \\
\hline $\boldsymbol{\gamma}$ & $3.47 \pm 3.02$ & 8.0 & 0 & 4.50 & 5.25 \\
\hline $\boldsymbol{\delta}$ & $86.38 \pm 2.39$ & 9.0 & 85.0 & 87.0 & 87.0 \\
\hline
\end{tabular}

Note: Both immediate post-op and follow-up radiographs showed same values for angles $\alpha, \beta, \gamma$ and $\delta$.

Mean age of the patients involved in study was $65.6 \pm 4.7$ years. Fifty-four $(60 \%)$ patients were in age group of (61$70)$. Eighteen patients $(20 \%)$ were less than 60 years old and more than 70 years old each. Mean BMI of patients was $25.6 \pm 1.8 \mathrm{~kg} / \mathrm{m}^{2}$. Diagnosis was osteoarthritis of knee in all 90 patients.

Total number of male patients were 24 (26.66\%) and females were 66 (73.33). Six different functional scoring were done pre- and post-operatively for 111 knees. Mean, $\mathrm{SD}$ and $\mathrm{p}$ value was calculated for each scoring. Authors found significant difference ( $p$ value $\leq 0.01$ ) in pre- and post-operative values of VAS score, WOMAC osteoarthritic index, Oxford knee score, Bristol knee score, knee society objective knee score and Knee society functional score. This comparison of pre- and postoperative scores was done using Wilcoxon's sign rank test (Table 2).

Radiological outcome was recorded with the help of X-ray knee (anteroposterior and lateral views). Angles $\alpha, \beta, \gamma$ and $\delta$ were measured pre-operatively and post-operatively and compared. They were found to be same which suggest that there has been no change in position of implants. None of the patients had radiologic evidence of loosening of implants (Table 3) (Figure 2).

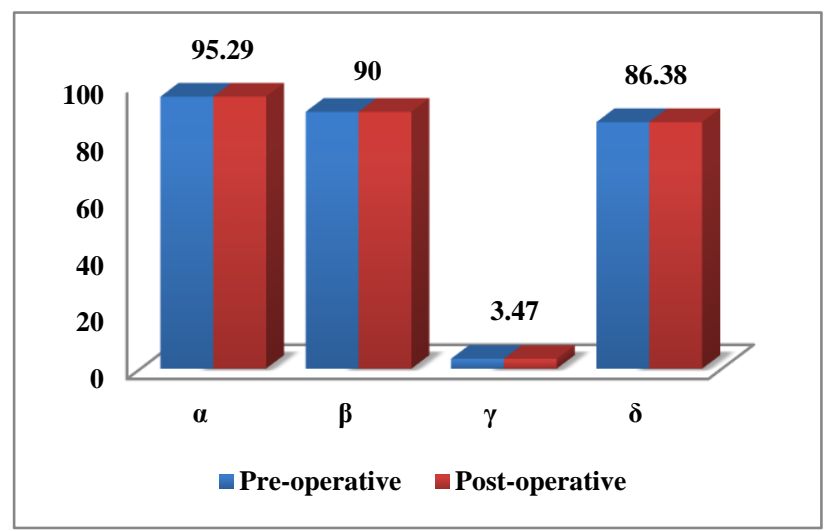

Figure 2: Comparison of mean values of above measured angles (immediate post-operative and at follow-up). 
No major complication was recorded in any post-operative patients. Six patients had developed superficial infection in early post-operative period which healed with daily wound care. The difference between pre-operative and post-operative, visual analogue scale score, WOMAC osteoarthritis index, Oxford knee score, Bristol knee score, knee society score is statistically significant with $\mathrm{p}<0.001$.

\section{DISCUSSION}

Longitudinal study was conducted in orthopedic outpatient department of tertiary care hospital in a metropolitan city of India during January 2012 to December 2018. Authors evaluated total 111 knees in 90 patients who underwent all polyethylene TKA. Functional and radiological evaluation was done by functional knee scores, VAS scores and Xray respectively.

Mean age of study participants in this study was 65.6 years. Mean BMI of study participants was $25.6 \mathrm{~kg} / \mathrm{m}^{2}$. APT components were reserved for elderly patient population, as few earlier studies reported excellent survivorship rates and functional outcomes in elderly patients with reduced physical demand. ${ }^{12,13}$ As an alternative to newer technologies which don't have a track record of clinical success, recent studies have advocated APT as a cost-effective option in younger, obese, higher activity patients. Ranawat et al reported combined knee society scores of greater than 170 for $95 \%$ of the patients at 5-year follow-up in patients under the age of 60 years undergoing APT TKA with a mean BMI of $29.2 \mathrm{~kg} / \mathrm{m}^{2}{ }^{13}$ Dalury et al reported favorable failure rates for APT components in obese patients (mean BMI of $34 \mathrm{~kg} / \mathrm{m}^{2}$ ) with low physical demand at 5 year follow-up. ${ }^{14}$ Also a longitudinal study conducted by Toman et al reported that APT components performed equivalent to MBT components for patients with BMI less than $37.5 \mathrm{~kg} / \mathrm{m}^{2}$ while studying the survivorship of APT versus MBT components. ${ }^{15}$ Few prospective studies recently reported that, APT components performed equivalently in both obese and non-obese patient groups, as well as younger and older patients. ${ }^{6,16}$ However, for patients with a BMI more than $40 \mathrm{~kg} / \mathrm{m}^{2}$, survivorship data of APT components is not validated by any of the current studies.

Authors found statistically significant improvement between the pre-operative and follow-up functional knee scores and VAS scores. There is paucity of literature with follow-up studies comparing functional and VAS scores of APT TKA at medium term follow-up. APT implants have been compared with MBT implants in many studies, which show modern APT implants are better or at least equal to MBT components in terms of survivorship, range of motion, complication rate and revision rate at 10 years follow-up..$^{5-9,12,15,17-21}$ APT components were also found to have a significantly lower rate of instability, tibial component loosening, peri-prosthetic fracture and infection. ${ }^{16}$ Few recent meta-analysis reported that results of APT component were comparable with or better than the MBT component in TKA. ${ }^{7,22}$ In a randomized clinical trial comparing APT to MBT components, Kalisvaart et al reported no statistical difference in knee ROM, stair climbing scores, implant durability and fixation at 5 years. ${ }^{23}$ Five year survivorship was $98.7 \%$ for the APT, $97.4 \%$ for MBT. A retrospective study which analyzed 27,657 TKAs, found 50\% lower risk of early revision for patients with APT. ${ }^{24}$ Also a study that analyzed 27,733 knee replacements in Swedish TKA registry with a followup of 4.5 years, reported superiority of APT over MBT in terms of implant survival. ${ }^{5}$

Authors found that there was no change in the TKA component alignment. This was measured on X-ray as $\alpha$, $\beta, \gamma$ and $\delta$ angles pre-operatively and post-operatively. These angles were found similar in immediate postoperative and 5 year follow up X-ray. None of the patients had radiological evidence of loosening or change in implant position at 5-year follow-up. Radio stereometric analysis (RSA) has been shown to predict the risk of future aseptic loosening on the basis of initial implant migration because of its extremely high resolution. If APT component shows no migration between one and two years after TKA, it has positive prognostic importance with regard to predicting future aseptic loosening. ${ }^{25} \mathrm{~A}$ randomized clinical study reported that, there was no difference in implant loosening and migration of both APT and MBT components on RSA, with favorable implant migration distance of APTs at 2 years. ${ }^{25,26}$ Also a prospective randomized study found that APT and MBT components were radiographically equivalent in terms of implant migration and loosening rates, with a little advantage to APT components. ${ }^{10}$ Gustke et al reported no revision for aseptic loosening and extremely low incidence of radiolucent lines in APT at 6 years follow-up. ${ }^{27}$

APT components have several advantages in the surgical preparation of the tibia. Thicker polyethylene insert can be used with the same amount of tibial resection, thus increasing the life of the prosthesis. Also, to achieve the same poly thickness as with an MBT, less tibial resection will be required leading to availability of larger metaphyseal surface area. This allows use of larger tibial component sizes, reducing the contact stresses transmitted across the joint and also preserves metaphyseal bone stock. It is suggested that a biomechanically sound construct with a polyethylene thickness of between 8 to $10 \mathrm{~mm}$ is more easily achieved by using an APT component. ${ }^{28}$

One retrospective study, reported higher risk of revision due to infection in MBT components than for APT components.5 In MBT components, polyethylene debris are liberated due to micro-motion at the liner-tray interface despite of security of the liner-capture mechanism. These debris cause synovitis, hyperemia, effusion and an impaired immune response making MBT components vulnerable for the development of periprosthetic joint infection. ${ }^{29}$

Few drawbacks of APT components were described by studies with finite element analysis that demonstrate APT 
components generate higher stresses on the proximal tibial and greater micro motion that can result in component loosening and tibial bone collapse in patients with higher demand. The MBT components have demonstrated better biomechanical performance during a squat activity compared to the APT. While selecting appropriate components for patient, above results should be considered. ${ }^{30}$

\section{CONCLUSION}

Despite multiple studies proving similar or better results in APT components compared to MBT components at minimum 5-year follow-up, most orthopedic surgeons are still reluctant to use APT components for younger, obese and higher demand patients. Authors conclude that APT components have good to excellent functional outcome and good radiological outcome at 5 years. APT components are safe in view of radiologic component migration at 5-year follow-up. Authors are of the opinion that APT are better, safe and cheaper option for TKA in patients with lower demand of activity and don't have complex knee deformity.

Funding: No funding sources

Conflict of interest: None declared

Ethical approval: The study was approved by the institutional ethics committee

\section{REFERENCES}

1. Gioe TJ, Maheshwari AV. The all-polyethylene tibial component in primary total knee arthroplasty. J Bone Jt Surg - Ser A. 2010;92:478-87.

2. Hamilton LR. UCI total knee replacement. A followup study. J Bone Joint Surg Am. 1982;64:740-4.

3. Ducheyne P, Kagan A, Lacey JA. Failure of total knee arthroplasty due to loosening and deformation of the tibial component. J Bone Joint Surg Am. 1978;60:384-91.

4. Evanski PM, Waugh TR, Orofino CF, Anzel SH. UCI knee replacement. Clin Orthop Relat Res. 1976:33-8.

5. Gudnason A, Hailer N, Annette W, JBJS MS-, 2014 undefined. All-polyethylene versus metal-backed tibial components-an analysis of 27,733 cruciateretaining total knee replacements from the Swedish knee arthroplasty register. J Lww Com. 2014:994-9.

6. Kremers HM, Sierra RJ, Schleck CD, Berry DJ, Cabanela ME, Hanssen AD, et al. Comparative survivorship of different tibial designs in primary total knee arthroplasty. J Bone Jt Surg - Am. 2014;96:e121.

7. Nouta KA, Verra WC, Pijls BG, Schoones JW, Nelissen RGHH. All-polyethylene tibial components are equal to metal-backed components: Systematic review and meta-regression. Clin Orthop Relat Res. 2012;470:3549-59.

8. van der Ven A, Scott RD, Barnes CL. Allpolyethylene tibial components in octogenarians: survivorship, performance, and cost. Am J Orthop (Belle Mead NJ). 2014;43:21-4.

9. Bettinson KA, Pinder IM, Moran CG, Weir DJ, Lingard EA. All-polyethylene compared with metalbacked tibial components in total knee arthroplasty at ten years: A prospective, randomized controlled trial. J Bone Jt Surg - Ser A. 2009;91:1587-94.

10. Norgren B, Dalén T, Nilsson K. All-poly tibial component better than metal-backed: a randomized RSA study. Knee. 2004;11:189-96.

11. Kilincoglu V, Unay K, Akan K, Esenkaya I, Poyanli O. Component alignment in simultaneous bilateral or unilateral total knee arthroplasty. Int Orthop. 2011;35:43-6..

12. Pagnano MW, Levy BA, Berry DJ. Cemented all polyethylene tibial components in patients age 75 years and older. Clin Orthop Relat Res. 1999;367:7380.

13. Ranawat AS, Mohanty SS, Goldsmith SE, Rasquinha VJ, Rodriguez JA, Ranawat CS. Experience with an all-polyethylene total knee arthroplasty in younger, active patients with follow-up from 2 to 11 years. J Arthroplasty. 2005;20:7-11.

14. Dalury DF, Tucker KK, Kelley TC. All-polyethylene tibial components in obese patients are associated with low failure at midterm follow-up. Clin Orthop Relat Res. 2012;470:117-24.

15. Toman J, Iorio R, Healy WL. All-polyethylene and metal-backed tibial components are equivalent with BMI of less than 37.5. Clin Orthop Relat Res. 2012;470:108-16.

16. Houdek MT, Wagner ER, Wyles CC, Watts CD, Cass JR, Trousdale RT. All-polyethylene tibial components: an analysis of long-term outcomes and infection. J Arthroplasty. 2016;31:1476-82.

17. Gioe TJ, Sinner P, Mehle S, Ma W, Killeen KK. Excellent survival of all-polyethylene tibial components in a community joint registry. Clin Orthop Relat Res. 2007;464:88-92.

18. Pomeroy DL, Schaper LA, Badenhausen WE, Suthers KE, Smith MW, Empson JA, et al. Results of all-polyethylene tibial components as a cost-saving technique. Clin Orthop Relat Res. 2000;380:140-3.

19. Apel DM, Tozzi JM, Dorr LD. Clinical comparison of all-polyethylene and metal-backed tibial components in total knee arthroplasty. Clin Orthop Relat Res. 1991;273:243-52.

20. Rand JA. Comparison of metal-backed and allpolyethylene tibial components in cruciate condylar total knee arthroplasty. J Arthroplasty. 1993;8:307-13.

21. Voigt J, Mosier M. Cemented all-polyethylene and metal-backed polyethylene tibial components used for primary total knee arthroplasty. J Bone Jt Surgery-American. 2011;93:1790-8.

22. Cheng T, Zhang G, Zhang X. Metal-backed versus all-polyethylene tibial components in primary total knee arthroplasty: a meta-analysis and systematic review of randomized controlled trials. Acta Orthop. 2011;82:589-95. 
23. Kalisvaart MM, Pagnano MW, Trousdale RT, Stuart MJ, Hanssen AD. Detectable differences at five years. J Bone Joint Surg Am. 2012;94(6):481-9.

24. Mohan V, Inacio MCS, Namba RS, Sheth D, Paxton EW. Monoblock all-polyethylene tibial components have a lower risk of early revision than metal-backed modular components. Acta Orthop. 2013;84:530-6.

25. Adalberth G, Nilsson KG, Byström S, Kolstad K, Milbrink J. All-polyethylene versus metal-backed and stemmed tibial components in cemented total knee arthroplasty. A prospective, randomised RSA study. J Bone Joint Surg Br. 2001;83:825-31.

26. Hyldahl H, Regnér L, Carlsson L, Kärrholm J, Weidenhielm L. All-polyethylene vs. metal-backed tibial component in total knee arthroplasty-a randomized RSA study comparing early fixation of horizontally and completely cemented tibial components: part 1. Horizontally cemented components: AP better fixated than MB. Acta Orthop. 2005;76:769-77.

27. Gustke K, Gelbke M. All-polyethylene tibia component use for elderly, low demand total knee replacement patients. J Arthroplasty. 2017;32(8):2421-6.

28. Bartel DL, Rawlinson JJ, Burstein AH, Ranawat CS, Flynn WF. Stresses in polyethylene components of contemporary total knee replacements. Clin Orthop Relat Res. 1995;317:76-82.

29. Petrie RS, Hanssen AD, Osmon DR, Ilstrup D. Metal-backed patellar component failure in total knee arthroplasty: a possible risk for late infection. Am J Orthop (Belle Mead NJ). 1998;27:172-6.

30. Brihault J, Navacchia A, Pianigiani S, Labey L, De Corte R, Pascale V, et al. All-polyethylene tibial components generate higher stress and micromotions than metal-backed tibial components in total knee arthroplasty. Knee Surg Sport Traumatol Arthrosc. 2016;24:2550-9.

Cite this article as: Patel D, Shah KA, Desai MM, Shah SS. All polyethylene tibia - results of functional and radiological outcome at 5 years follow-up. Int J Res Orthop 2020;6:945-50. 